\title{
Primary Response Assessment Method for Concept Design of Monotonous Thin-Walled Structures
}

\author{
V. Zanic, P. Prebeg
}

A concept design methodology for monotonous, tapered thin-walled structures (wing/fuselage/ship/bridge) is presented including modules for: model generation; loads; primary (longitudinal) and secondary (transverse) strength calculations; structural feasibility (buckling/fatigue/ultimate strength criteria); design optimization modules based on ES/GA/FFE; graphics. A method for primary strength calculation is presented in detail. It provides the dominant response field for design feasibility assessment. Bending and torsion of the structure are modelled with the accuracy required for concept design. A '2.5D-FEM' model is developed by coupling a 1D-FEM model along the 'monotonity' axis and a 2D-FEM model(s) transverse to it. The shear flow and stiffness characteristics of the cross-section for bending and pure/restrained torsion are given, based upon the warping field of the cross-section. Examples: aircraft wing and ship hull.

Keywords: thin-walled structures, shear flow, FEM, concept design.

\section{Introduction}

The concept design methodology for monotonous, tapered, thin-walled structures (wing/fuselage/ship/bridge) is presented. The problem solution is based on the OCTOPUS program $[1,2]$. It contains: (A) response and feasibility analysis modules (FIN-CREST), (B) decision making-synthesis modules (DeMak) and (C) interaction/visualization programs (MAESTRO MM/MG and DeVIEW) that irerate in the design cycle. The modules are summarized in Table 1 as modules la-8c.

(A) The analytical (CREST) modules and methods are fully described in [2]. Module-1a INDAT is used for data generation, combined with the MAESTRO FEM MODELER [8]. Module-2 LOAD is used for design load generation. Module-1b MIND is used for determining the minimal scantlings based on prescribed rules (LR, DnV, ABS, etc.) Module-3a LTOR is used for direct calculation of the primary strength (shear flow and corrected stresses in bending and warping torsion). They are calculated using an original extended beam theory[5, 6]. Module-3b TOKV is used for the transverse strength calculation (newly developed 8-node stiffened panel macro-elements are used for modeling the transverse structural response). Module- 4 is the PANEL library of structural serviceability and ultimate strength criteria for the structural adequacy calculation [4], using the response fields generated in modules $3 \mathrm{a}$ and $3 \mathrm{~b}$. Module- $8 \mathrm{a}$ is VB-SHELL for the designer-model interaction. Module-8b MAESTRO GRAPHIC [8] is used for presenting the model (loading, response, adequacy, etc.)

(B) Synthesis (DeMak) modules and methods are documented in $[1,3]$. Local variables for substructures $(\mathrm{s}=1, \ldots, \mathrm{NS})$, are denoted $\underline{\mathbf{x}}^{\mathrm{s}}=\left\{\underline{\mathrm{x}}_{\mathrm{i}}\right\}^{\mathrm{s}}=\left\{\mathrm{t}_{\text {plating }}, \mathrm{n}_{\text {stiffeners }}, \mathrm{h}_{\text {web }}\right.$ ... $\}^{\mathrm{s}}$. Substructure areas $\mathbf{X}=\left\{\mathrm{X}_{\mathrm{s}}\right\}$ are intermediate (global) variables, where $\mathrm{X}_{\mathrm{s}}=\mathrm{X}_{\mathrm{s}}\left(\underline{\mathbf{x}}^{\mathbf{s}}\right)$. Project $\mathrm{k}$ is defined as $\mathbf{P}^{\mathbf{k}}=\left\{\underline{\mathbf{x}}^{1}\right.$, $\left.\ldots, \underline{\mathbf{x}}^{\mathrm{NS}}, \mathbf{x}_{\text {fixed }}\right\}^{\mathrm{k}}$. Design criteria (attributes, objectives, constraints) are formulated as a library of mathematical func- tions/procedures for driving the optimization process or feasibility check. OCTOPUS metamodeling of failure surfaces is based on the most unsatisfied constraint from each local problem. They are added to the set of global constraints. The value function for a global level is a multicriterion combination of normalized attribute functions. The solution strategy involves generation of designs, using (a) a Random Number Generator in the first cycles of design space exploration, and (b) Fractional Factorial Experiments for subsequent cycles. Coordination is performed by modifying $\mathrm{v}\left(\underline{\mathbf{x}}^{\mathbf{S}}\right)$ respective to its divergence from globally optimal substructure area $\mathrm{X}^{\mathrm{s}}$. Special provisions:

generation of promising designs using 27 designs obtained from Orthogonal Array L27.

- extensive usage of tables of optimized profiles to speed up the generation process.

Modules 6 and 7c (GAZ) are used for calculating the sensitivity of the structural response with respect to the design variables, based on the global strength module FIN/LTOR [7]. Module-7a GLO is used for global level MODM optimization (level 1) of the cross-section. Module-7b LOC is used for local coordinated MADM decision making via sequential application of stochastic search methods and Theory of experiments.

(C) Module- $8 \mathrm{c}$ DeVIEW is used for the designer-model interaction with graphic presentation of designs in the design and attribute spaces. The stratified distances from the target (or the ideal) design, calculated by $\mathrm{L}_{\mathrm{p}}$ metric are used as a means of visualizing the multidimensional space of the design attributes and/or free variables. Visualization is the most powerful tool for the designer's understanding of the Decision Support Problem. It generates expert knowledge about the problem for all participants involved, and helps the designer to identify advantageous combinations of variables, feasible options and clusters of non-dominated designs (Pareto frontier, thus enabling realistic decision support to the head and structural designer). 
Table 1: Summary of OCTOPUS modules

\begin{tabular}{|c|c|}
\hline MODULE & OCTOPUS \\
\hline (1a) STRUCTURAL MODEL & $\begin{array}{l}\text { MAESTRO files generated by program MM and used in OCTOPUS } \\
\text { (s/r INDAT) }\end{array}$ \\
\hline (2) LOAD MODEL & $\begin{array}{l}\text { Rule Loads + designer given loads generated } \\
\text { automatically by OCTOPUS s/ r LOAD }\end{array}$ \\
\hline (1b) MINIMAL DIMENSIONS & Minimal dimensions by OCTOPUS s/r MIND \\
\hline $\begin{array}{l}\text { (3a) RESPONSE CALCULATIONS - } \\
\text { - PRIMARY STRENGTH } \\
\text { (u-displ.; stresses } \sigma \mathrm{X}, \tau \text { ) }\end{array}$ & $\begin{array}{l}\text { Extended beam theory (cross section warping fields in bending and } \\
\text { torsion, normal stresse s, respective shear flows) } \\
\text { program LTOR }\end{array}$ \\
\hline $\begin{array}{l}\text { (3b) RESPONSE CALCULATION - } \\
\text {-TRANSVERSE STRENGTH } \\
\text { (displacements v, w, } \theta x \text { stresses } \sigma \mathrm{y} \text { ) }\end{array}$ & $\begin{array}{l}\text { FEM calculation using beam element with or without rigid ends and } \\
\text { stiffened panel macroelements } \\
\text { program TOKV }\end{array}$ \\
\hline $\begin{array}{l}\text { (4) FEASIBILITY CALCULATION } \\
\qquad g_{i}=\frac{C_{i}-D_{i}}{C_{i}+D_{i}} \quad \text { (Normalized Safety Factor) }\end{array}$ & $\begin{array}{l}\text { Calculation of macroelement feasibility using library of safety criteria } \\
\text { in program PANEL (C - capability; D - Demand from 3a and 3b) }\end{array}$ \\
\hline $\begin{array}{l}\text { (5) RELIABILITY CALCULATION } \\
\text { (not used) }\end{array}$ & $\begin{array}{l}\text { FORM approach to panel reliability. } \\
\text { Upper Dietlevsen bound as design attribute }\end{array}$ \\
\hline $\begin{array}{l}\text { (6) DECISION SUPPORT PROBLEM DEFINITION } \\
\text { (interactive) }\end{array}$ & $\begin{array}{l}\text { Constraints: } \\
\text { User given Minimal dimensions Library of criteria (see 4) } \\
\text { Objectives: minimal weight, minimal cost, maximal safety }\end{array}$ \\
\hline (7a, b, c) OPTIMIZATION METHOD & $\begin{array}{l}\text { Decision making procedure using } \\
\text { a) Global MODM optimization program GLO } \\
\text { b) Local MADM optimization module LOC } \\
\text { c) Coordination module GAZ }\end{array}$ \\
\hline (8a, b, c) PRESENTATION OF RESULTS & a) VB Environment, b) Program MG, c) DeVIEW graphic tool \\
\hline
\end{tabular}

\section{Modeling philosophy for primary response in concept design}

Classical FE modeling, giving good insight into stresses and deformations, is not capable of giving the efficient and fast answers regarding feasibility criteria (buckling, fatigue, yield) required by the Rules. However, structural feasibility and compliance with the Rule requirements are of primary interest, not stresses or deformations.

Most of the local failure criteria, e.g. various buckling failure modes of stiffened panels, require specified force and displacement boundary conditions. They are available only if logical structural parts, such as complete stiffened panels between girders and frames, are modeled (macro-elements).
For the concept design structural evaluation of the primary response (longitudinal strength, torsional strength), the beam idealization of a wing/ship/bridge is often used. A primary strength calculation provides the dominant response field (Demand) for design feasibility assessment. The evaluation is based on extended beam theory, which needs cross-sectional characteristics. These are obtained using analytical methods, which can be very complicated for real combinations of open and closed cross-sections.

Application of energy based numerical methods gives an opportunity for an alternative approach to the given problems. The method is based on decomposing a cross-section into the line finite elements between nodes $i$ and $j$ with coordinates $\left(y_{i}, z_{i}\right),\left(y_{j}, z_{j}\right)$; element thickness $t^{e}$; material characteristics (Young's modulus $E$ /shear modulus $G$ ); material
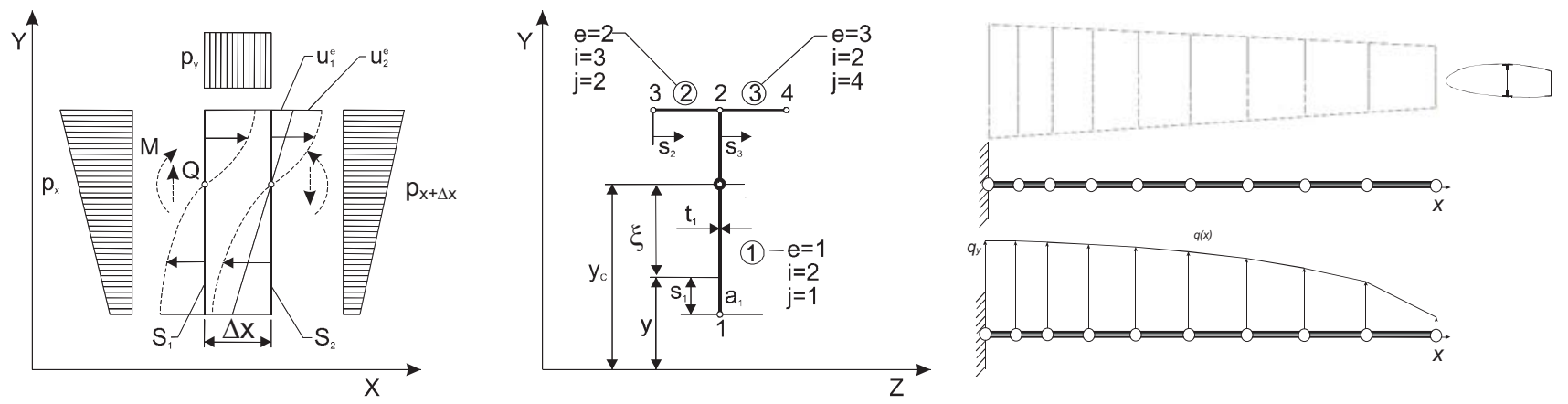

Fig. 1: Transverse strip (S1-S2) with external loading $\mathrm{p}$, warping fields $\mathrm{u}$ and 1D/2D FEM idealization 


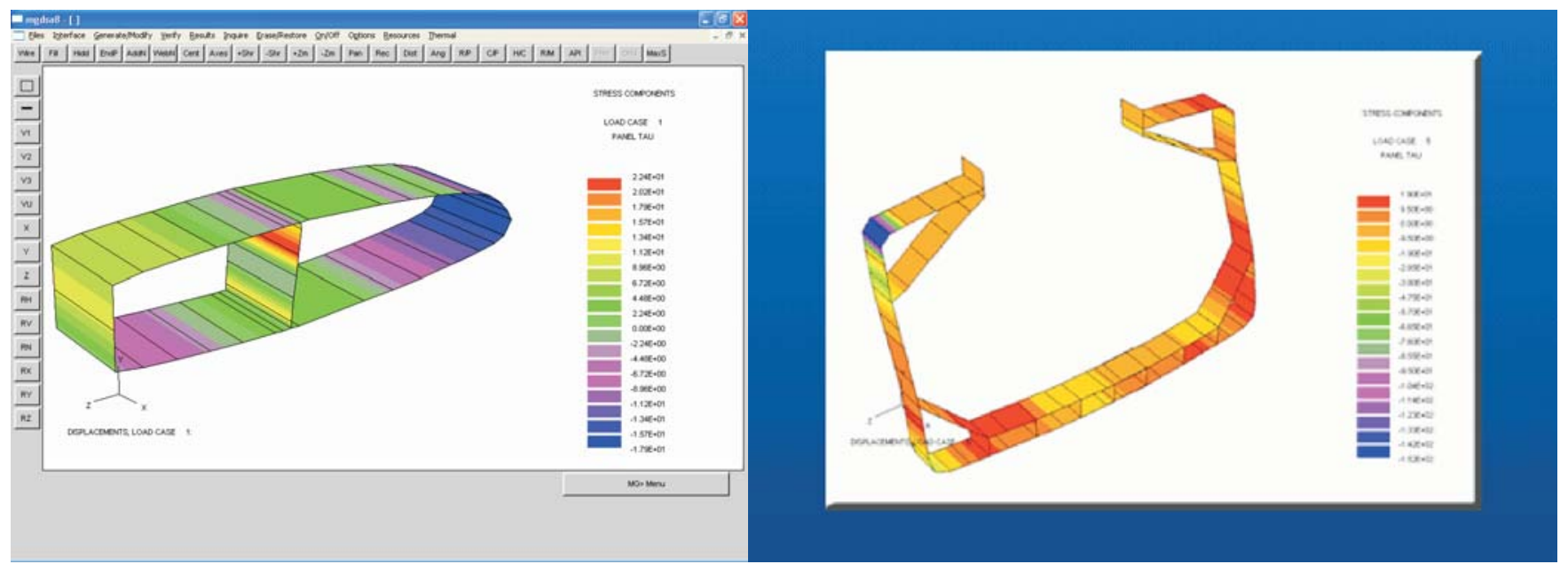

Fig. 2: OCTOPUS/MAESTRO GRAPHIC shear stress fields in wing and bulk-carrier transverse strip

efficiency $R N$ and $R S$ (due to cutouts, lightening holes, etc.) with respect to normal/shear stresses.

Using the FEM approach, a procedure is developed for calculating the set of cross-sectional geometric and stiffness characteristics at position $\mathrm{x}$ denoted $\mathbf{G}^{\mathbf{X}}$ with the following elements:

- Cross-section area $A$

- Center of gravity $Y_{\mathrm{CG}}, Z_{\mathrm{CG}}$,

- Shear/torsion center $Y_{\mathrm{CT}}, Z_{\mathrm{CT}}$.

- Moments of inertia with respect to the center of gravity: $I_{Y}$, $I_{Z}, I_{y z}, I_{p}$; principal: $I_{1}, I_{2}, \phi_{0}$-angle of axis- 1 w.r.t. Z-axis,

- Horizontal and vertical bending:

- Flexural stiffness $E I_{Z}, E I_{Y}$

- Shear stiffness $G A_{\mathrm{V}}, G A_{\mathrm{H}}$,

- Cross-section axial stiffness $E A$

- Torsional stiffness $G I_{\mathrm{T}}$

- Warping stiffness $E I_{\mathrm{W}}$.

Standard stiffness matrices (alternatively with geometrical nonlinearity [4]), for axial $\left(\mathbf{k}_{\mathrm{u}}\right)$ and flexural $\left(\mathbf{k}_{\mathrm{v}}, \mathbf{k}_{\mathrm{w}}\right)$ and torsional response modes are given as functions of the geometric set $\mathbf{G}^{\mathbf{x}}$ :

$\mathbf{k}_{\mathrm{u}}=\frac{A E}{L} \cdot\left[\begin{array}{cc}1 & -1 \\ -1 & 1\end{array}\right]$;

$\mathbf{k}_{\mathrm{v}}=\frac{E I_{Z}}{\left(1+\Phi_{Y}\right) L^{3}}\left[\begin{array}{cccc}12 & & & \text { Symm } \\ 6 L & \left(4+\Phi_{Y}\right) L^{2} & & \\ -12 & -6 L & 12 & \\ 6 L & \left(2-\Phi_{Y}\right) L^{2} & -6 L & \left(4+\Phi_{Y}\right) L^{2}\end{array}\right]$;

$\Phi_{Y}=\frac{12 E I_{z}}{L^{2} \cdot G A_{Y}}$,

$\mathbf{k}_{\mathrm{W}}$ is obtained similarily. Stiffness matrices for free torsion $\left(\mathbf{k}_{\mathrm{T}}\right)$ and restrained warping $\left(\mathbf{k}_{\omega}\right)$ read:

$\mathbf{k}_{\mathrm{T}}=\frac{G I_{\mathrm{T}}}{L}\left[\begin{array}{cccc}6 / 5 & & & \text { Symm } \\ L / 10 & 2 L^{2} / 15 & & \\ -6 / 5 & L / 10 & 6 / 5 & \\ L / 10 & -2 L^{2} / 30 & -L / 10 & 2 L^{2} / 15\end{array}\right]$,
$\mathbf{k}_{\omega}=\frac{E I_{\mathrm{W}}}{L^{3}}\left[\begin{array}{cccc}12 & & & \text { Symm } \\ 6 L & 4 L^{2} & & \\ -12 & -6 L & 12 & \\ 6 L & 2 L^{2} & -6 L & 4 L^{2}\end{array}\right]$.

Global stifness matrix $\mathbf{K}_{\mathbf{1 D}}$ is obtained by the combination of modal stiffnesses corrected for centroid and shear centre position relative to the position of the origin of global C.S.

$a_{L}=\left\{\begin{array}{c}u \\ v_{\mathrm{T}} \\ w_{\mathrm{T}} \\ \theta \\ \theta_{y \mathrm{~T}} \\ \theta_{z \mathrm{~T}} \\ \theta_{, x}\end{array}\right\}$

$=\left[\begin{array}{ccccccc}1 & 0 & 0 & \omega_{0} & z_{\mathrm{CG}} \lambda+y_{\mathrm{CG}} \mu & z_{\mathrm{CG}} \mu-y_{\mathrm{CG}} \lambda & 0 \\ 0 & \lambda & \mu & z_{\mathrm{CS}} & 0 & 0 & 0 \\ 0 & -\mu & \lambda & -y_{\mathrm{CS}} & 0 & 0 & 0 \\ 0 & 0 & 0 & 1 & 0 & 0 & 0 \\ 0 & 0 & 0 & 0 & \lambda & -\mu & -y_{\mathrm{CS}} \\ 0 & 0 & 0 & 0 & \mu & \lambda & z_{\mathrm{CS}} \\ 0 & 0 & 0 & 0 & 0 & 0 & 1\end{array}\right]\left\{\begin{array}{c}U \\ V \\ W \\ \theta \\ \Theta_{y} \\ \Theta_{z} \\ \Theta_{, x}\end{array}\right\}$

$=\mathbf{t} \mathbf{a}_{\mathrm{G}}$

$\mathbf{f}_{\mathrm{L}}=\left\{\begin{array}{c}\mathbf{f}_{\mathrm{u}} \\ \mathbf{f}_{\mathrm{v}} \\ \mathbf{f}_{\mathrm{w}} \\ \mathbf{f}_{\theta}\end{array}\right\}=\left\{\begin{array}{cccc}\mathbf{k}_{\mathrm{u}} & 0 & 0 & 0 \\ 0 & \mathbf{k}_{\mathrm{v}} & 0 & 0 \\ 0 & 0 & \mathbf{k}_{\mathrm{w}} & 0 \\ 0 & 0 & 0 & \mathbf{k}_{\mathrm{T}}+\mathbf{k}_{\omega}\end{array}\right\}\left\{\begin{array}{c}\mathbf{a}_{\mathrm{u}} \\ \mathbf{a}_{\mathrm{vB}} \\ \mathbf{a}_{\mathrm{wB}} \\ \mathbf{a}_{\theta}\end{array}\right\}=\mathbf{k}_{\mathrm{L}}^{e} \cdot \mathbf{a}_{\mathrm{L}}$

where

$\mathbf{a}_{\mathrm{L}}=\mathbf{T} \mathbf{a}_{\mathrm{G}}, \quad \mathbf{T}=\left[\begin{array}{ll}\mathbf{t} & 0 \\ 0 & \mathbf{t}\end{array}\right]$.

Finally, the global stiffness matrix $\mathbf{K}_{\mathbf{1 D}}$ is obtained as the sum of the element stiffness matrices $\mathbf{k}_{\mathrm{G}}^{e}=\mathbf{T}^{T} \mathbf{k}_{\mathrm{L}}^{e} \mathbf{T}$ with appropriate node numbering. The system $\mathbf{K}_{\mathbf{1 D}} \mathbf{a}=\mathbf{F}$ can now be solved for unknown displacements a which in turn enable determination of element parameters $\mathbf{a}_{\mathrm{L}}$. From these parame- 
ters the element axial, bending and torsion parameter distributions, based on the applied shape functions, can be derived (e.g. $\theta(x), \theta_{, x}(x), \theta_{, x x}(x), \theta_{, x x x}(x)$ for torsion). The key element for calculation of the response of the complex thinwalled structure is therefore determination of elements of $\mathbf{G}^{\mathrm{x}}$. A simple and elegant FEM procedure for such a calculation is presented in the sequel.

\section{Calculation of response for a transverse strip with a complex cross section}

The shear flow and geometrical characteristics of the cross section in bending and torsion is usually calculated using analytical methods. Such calculations become rather complicated for multiple-connected cross section graphs with a combination of open and closed (cell) contours. Application of numerical methods based on the energy approach offers an elegant alternative. The procedure is based on section decomposition into finite elements, as first introduced by Herman and Kawai. In the sequel, the method of calculation as described in $[5,6]$ is presented. It has been successfully used in practical calculations since its development for [10]. The simplest decomposition of thin-walled cross-section (symmetric or not) into line finite elements (segments) is shown in Figs. 1 and 2. These elements form stiffened panel macro-elements for the feasibility evaluation.

The methodology is based on applying the principle of minimum total potential energy (П) with respect to parameters which define the displacement fields of the structure. The primary displacement field (following classical beam theory) is defined via displacements and rotations of the cross section as a whole. Secondary displacement field $\mathbf{u}_{2}(x, y, z) \equiv \mathbf{u}(x, y, z)$ represents warping (deplanation) of the cross section. For piecewise-linear FEM idealization of the cross-section, divided into $n$ elements, with shape functions $\mathbf{N}$ in the element coordinate system $(\mathrm{x}, \mathrm{s})$, the warping field reads:

$u(s)_{x=x_{0}}^{e}=\mathbf{N}^{\mathrm{T}} \cdot \mathbf{u}^{e}=\left\{\begin{array}{ll}1-\frac{s}{l^{e}} & \frac{s}{l^{e}}\end{array}\right\}\left\{\begin{array}{l}u_{i} \\ u_{j}\end{array}\right\}$.

Element strain and stress fields $\epsilon$ and $\sigma$ are obtained from the strain-displacement and stress-strain relations:

$$
\begin{gathered}
\varepsilon \rightarrow \gamma_{x s}=\frac{\partial u}{\partial s}=\mathbf{B}^{\mathrm{T}} \cdot \mathbf{u}^{e}=\left\{-\frac{1}{l^{e}} \frac{1}{l^{e}}\right\}\left\{\begin{array}{l}
u_{i} \\
u_{j}
\end{array}\right\}, \text { and } \\
\sigma \rightarrow \tau_{x s}=G^{e} \gamma_{x s}=G^{e} \mathbf{B}^{\mathrm{T}} \cdot \mathbf{u}^{e} .
\end{gathered}
$$

The total potential energy of the $\Delta x$-long transverse strip of the beam, with the cross-section divided into $n$ elements, reads:

$$
\begin{aligned}
\Pi & =\sum_{n}\left[\int_{V^{e}} \sigma^{T} \varepsilon \mathrm{d} V-\int_{S^{e}} p(x, s) u(s) \mathrm{d} S\right] \\
& =\sum_{e}\left[\int_{V^{e}} \frac{1}{2} \mathbf{B}^{\mathrm{T}} \mathbf{B} \mathrm{d} V-\int_{S^{e}} F(s) u(s) \mathrm{d} S\right] \\
& =\Delta x \sum_{e}\left[\frac{1}{2} \mathbf{u}^{e \mathrm{~T}} \mathbf{K}^{e} \mathbf{u}^{e}-\mathbf{u}^{e \mathrm{~T}} \mathbf{F}^{e}\right],
\end{aligned}
$$

where $p(x, s)$ is the external loading on two cross sections $\left(S_{1}\right.$ and $S_{2}$ ) of the strip. Minimization of $\Pi$ leads to the classical FEM matrix relation $\mathbf{K}_{\mathbf{2 D}} \mathbf{u}_{\mathbf{2 D}}=\mathbf{F}_{\mathbf{2 D}}$ (shortened to $\mathbf{K} \mathbf{u}=\mathbf{F}$ ). The element stiffness matrix for the proposed linear displacement distribution along the line element (the same for bending and torsion) reads:

$$
\mathbf{K}^{e}=\frac{G^{e} \cdot t^{e} \cdot R S^{e}}{l^{e}}\left[\begin{array}{cc}
1 & -1 \\
-1 & 1
\end{array}\right],
$$

where $R S$ is the prescribed shear efficiency.

\section{Cross-sectional shear stress distribution due to bending}

In the case of bending, the net external load (due to bending moments $\mathrm{M}(\mathrm{x}+\Delta \mathrm{x})$ and $\mathrm{M}(\mathrm{x}))$ is the normal stresses:

$$
\begin{aligned}
p(x, s) & =\sigma(x+\Delta x, s)_{S 2}-\sigma(x, s)_{S 1}=\frac{\partial}{\partial x} \sigma(x, s) \Delta x \\
& =Q(x) \frac{\xi_{C}(s)}{I(x)} \Delta x,
\end{aligned}
$$

where $\xi_{C}(s)$ is distance from the point to N. A.

The load vector for a nonsymmetrical cross-section in, e.g., bending about the $z$ axis reads:

$$
\begin{aligned}
& \mathbf{F}_{z}^{e}(x)=Q_{y}(x) \cdot \overline{\mathbf{F}}_{z}^{e}(x)=\frac{E^{e} \cdot Q_{y}(x) \cdot t^{e} \cdot R N^{e}}{E\left(I_{Y} I_{Z}-I_{Y Z}^{2}\right)} \\
& {\left[I_{Y} \cdot\left\{\begin{array}{l}
\frac{y_{i \mathrm{C}} \cdot l^{e}}{2}+\frac{l^{e^{2}} \sin \alpha^{e}}{6} \\
\frac{y_{i \mathrm{C}} \cdot l^{e}}{2}+\frac{l^{e^{2}} \sin \alpha^{e}}{3}
\end{array}\right\}-I_{Y Z} \cdot\left\{\begin{array}{l}
\frac{z_{i \mathrm{C}} \cdot l^{e}}{2}+\frac{l^{e^{2}} \sin \alpha^{e}}{6} \\
\frac{z_{i \mathrm{C}} \cdot l^{e}}{2}+\frac{l^{e^{2}} \sin \alpha^{e}}{3}
\end{array}\right\}\right] .}
\end{aligned}
$$

For bending around the $Y$ and $Z$ axes, the matrix relations $\mathbf{K} \mathbf{u}=\mathbf{F}$ with $\mathbf{u}=Q(x) \cdot \overline{\mathbf{u}}$ can be converted into expressions for the warping due to unit load $\overline{\mathbf{F}}$. For node warping $u_{i}(x)$, unit warping $\overline{\mathbf{u}}(x)$ must be multiplied by $Q(x)$. This enables the assessment of shear stresses $\tau_{Y}^{e}$ or $\tau_{Z}^{e}$ from the expression $\tau^{e}=G\left(u_{2 j}-u_{2 i}\right) / l^{e}$ in each element $e$ between nodes $i$ and $j$. If necessary, it is possible to calculate shear stress distribution $\tau_{x s}^{e}(s)$ more accurately, from the mean stress $\tau_{x s}^{k e}$ obtained from FEM, and the contribution to each element calculated analytically $\left(\tau_{x s}^{e}(s)\right)_{u}=\left(\tau_{x s}^{k e}\right)_{u}-\tau_{1 u}^{k e}+\tau_{1 u}(s) u=y$ or $z$ from expression (for symmetrical section):

$$
\begin{aligned}
& \left(\tau_{x S}^{e}(s)\right)_{z}=Q_{z} \cdot\left[G^{e} \cdot \mathbf{B}^{T} \cdot \overline{\mathbf{u}}_{y}^{e}-\frac{E^{e} \cdot R N^{e}}{E I_{Y}}\right. \\
& \left.\cdot\left(\frac{l^{e}}{3}\left(z_{i \mathrm{C}}+\frac{1}{2} z_{y \mathrm{C}}\right)-z_{i \mathrm{C}} \cdot s-\left(z_{j \mathrm{C}}-z_{i \mathrm{C}}\right) \cdot \frac{s^{2}}{2 l^{e}}\right)\right] .
\end{aligned}
$$

In this case, the sectional characteristics and shear center are easily obtained. The shear/torsion center position reads: $Y_{S C}=\sum_{n}\left[\int_{0}^{l^{e}} \tau_{Z}^{e} t^{e} d_{\mathrm{C}}^{e} \mathrm{~d} s\right]_{Q_{Z}=1}, Z_{S C}=\sum_{n}\left[\int_{0}^{l^{e}} \tau_{Y}^{e} t^{e} d_{\mathrm{C}}^{e} \mathrm{~d} s\right]_{Q_{Y}=1}$, where $d_{\mathrm{C}}^{e}$ is the normal distance from the centroid to $e$.

The shear stiffness for bending about the $Y$ and $Z$ axes, $G A_{\mathrm{V}}, G A_{\mathrm{H}}$ reads: 
$G A_{\mathrm{V}}=\left[\sum_{e}\left[\int_{0}^{l^{e}} \frac{\left(\left(\tau_{x s}^{e}\right)_{y}\right)_{Q_{Y}=1}^{2}}{G^{e}} \cdot t^{e} \cdot \mathrm{d} s\right] \cdot R S^{e}\right]^{-1} ;$

$G A_{\mathrm{H}}=\left[\sum_{e}\left[\int_{0}^{l^{e}} \frac{\left(\left(\tau_{x s}^{e}\right)_{z}\right)_{Q_{Z}=1}^{2}}{G^{e}} \cdot t^{e} \cdot \mathrm{d} s\right] \cdot R S^{e}\right]^{-1}$.

\section{Corrected normal stresses due to the influence of shear (shear lag)}

The normal stress must be corrected for (a) stress arising from a longitudinal change of the warping field and (b) normal stress due to correcting bending moment $\left(\mathrm{M}^{\mathrm{c}}\right)$, compensating for the loss of cross section equilibrium:

(a) $\left(\sigma_{x}^{c}\right)_{y}^{i}=\mathbf{E} \varepsilon=E^{e}\left(\frac{\partial u}{\partial x}\right)_{y}^{i}=-E^{e} \cdot p_{z} \cdot\left(\bar{u}_{y}^{i}-\bar{u}_{y}^{c}\right) \cdot R N^{e}$;

(b) $M_{y}^{c}=\sum_{e} \int_{0}^{l^{e}}\left(\sigma_{x}^{c}(s)\right)_{y} \cdot z_{c}(s) \cdot t^{e} \mathrm{~d} s$.

The total normal stress correction in node $i$ reads:

$\left(\sigma_{x}^{c T}\right)_{y}^{i}=R N^{e}\left[\frac{I_{Z} \cdot z_{i \mathrm{C}}-I_{Y Z} \cdot y_{i \mathrm{C}}}{\mathrm{E}\left(I_{Y} I_{Z}-I_{Y Z}^{2}\right)} M_{Y}^{c} E^{e}-E^{e} p_{z}\left(\bar{u}_{y}^{i}-\bar{u}_{y}^{c}\right)\right]$.

The approximate value of normal stress for simultaneous bending about axes $y$ and $z$ for node $I$ reads:

$$
\begin{aligned}
\sigma_{x}^{i} & =\left[R N^{e} \frac{M_{Z}}{E I_{Z}} E^{e} \cdot y_{i \mathrm{C}}+\left(\sigma_{x}^{c T}\right)_{z}^{i}\right] \\
& +\left[R N^{e} \frac{M_{Y}}{E I_{Y}} E^{e} \cdot z_{i \mathrm{C}}+\left(\sigma_{x}^{c T}\right)_{y}^{i}\right] .
\end{aligned}
$$

\section{Calculation of warping and primary shear stresses due to pure torsion}

A transverse strip of a thin-walled beam of length $\Delta x$ is subjected to torsional loading. The displacement field of the middle line of thin walled elements can be expressed using the warping function $\bar{u}(s)_{t=0}$, rotation $v_{s}(x)_{t=0}$ around the centre of twist, twist rate $\left(\theta_{x, x}\right)$ and angle $\left(\theta_{x}\right)$ of the twist reads:

$$
\begin{aligned}
& u(x, s)=\bar{u}(s)_{t=0} \cdot \theta_{x, x}\left(x_{0}\right), \\
& v(x, s, t) \rightarrow v_{s}(x)_{t=0}=d_{\mathrm{T} t=0} \cdot \theta_{x}\left(x_{0}\right),
\end{aligned}
$$

where $d_{\mathrm{T}}$ is the normal distance from the element to the center of torsion. The strain (with $\varepsilon_{s} \approx 0$ ) and stress fields read:

$$
\begin{aligned}
& \boldsymbol{\varepsilon}=\left\{\begin{array}{c}
\varepsilon_{x} \\
\gamma_{x s}
\end{array}\right\}=\left\{\begin{array}{c}
\bar{u} \cdot \theta_{x, x x} \\
\left(\frac{\partial \bar{u}}{\partial s}+d_{\mathrm{T}}\right) \theta_{x, x}
\end{array}\right\}, \\
& \boldsymbol{\sigma}=\mathbf{E} \boldsymbol{\varepsilon}=\left\{\begin{array}{c}
E \cdot \bar{u} \cdot \theta_{x, x x} \\
G \cdot\left(\frac{\partial \bar{u}}{\partial s}+d_{\mathrm{T}}\right) \theta_{x, x}
\end{array}\right\} .
\end{aligned}
$$

The total potential energy of a section is given by the standard expression:

$$
\Pi=U-W=\int_{V} \frac{1}{2} \sigma^{\mathrm{T}} \varepsilon \mathrm{d} V-W .
$$

After summation of all elements and transformation of local element displacements $\overline{\mathbf{u}}^{\ell}=\mathbf{N}^{T} \cdot \overline{\mathbf{u}}^{\ell}$ and loads $\mathbf{F}^{\ell}$ into global displacements $\overline{\mathbf{u}}$ and loads $\mathbf{F}$ we get:

$U=\Delta x \cdot \theta_{x, x}^{2} \cdot\left(\frac{1}{2} \overline{\mathbf{u}}^{\mathrm{T}} \mathbf{K} \overline{\mathbf{u}}-\overline{\mathbf{u}}^{\mathrm{T}} \mathbf{F}+\frac{1}{2} \sum_{e} R S^{e} \cdot d_{\mathrm{T}}^{e^{2}} l^{e} t^{e} G^{e}\right)$.

Where $\mathbf{K}^{e}=R S^{e} \cdot G^{e} \cdot t^{e} \int_{0}^{l^{e}} \mathbf{B} \mathbf{B}^{\mathrm{T}} \mathrm{d} s$

and $\mathbf{F}^{e}=-R S^{e} \cdot G^{e} \cdot t^{e} \cdot d_{\mathrm{T}}^{e} \int_{0}^{l^{e}} \mathbf{B} \mathrm{d} s$.

Minimization of total potential energy leads to two sets of equations:

(1) $\delta \Pi_{\theta^{\prime}}=0(1 \mathrm{D}$ beam torsion $)$ and

(2) $\delta \Pi_{\bar{u}}=0$ (2D cross-section warping).

A second set of equations, $\delta \Pi_{u}=\delta U_{u}=0 \rightarrow \mathbf{K} \overline{\mathbf{u}}=\mathbf{F}$, enables determination of the unit warping field.

The primary shear stresses on the elements which are parts of closed contours (cc) and open sections (os) can now be calculated as functions of $1 \mathrm{D}$ twist rates $\theta_{, x}(\mathrm{x})$ (to be obtained from the first relation for $1 \mathrm{D}$ beam torsion):

$\tau_{x s}^{k e^{(\mathrm{cc})}}=G^{e} \cdot \theta_{x, x}\left(\left\{\begin{array}{ll}-\frac{1}{l^{e}} & \frac{1}{l^{e}}\end{array}\right\}\left\{\begin{array}{c}\bar{u}_{i} \\ \bar{u}_{j}\end{array}\right\}+d_{\mathrm{T}}^{e}\right)$ and

$\tau_{x s \max }^{k e^{(\mathrm{os})}}=G^{e} \cdot \theta_{x, x} \frac{t^{e}}{2}$

\section{Calculation of torsional and warping stiffness of thin-walled structures}

To solve the equation for $1 \mathrm{D}$ beam free torsion, the torsional stiffness of elements which are parts of the open $e_{\mathrm{O}}$ and closed cells $e_{\mathrm{c}}$ can now be calculated using the known unit warping field $\overline{\mathbf{u}}$ :

$G I_{\mathrm{To}}=\sum_{e_{\mathrm{o}}} G^{e} \frac{l^{e} t^{e^{3}}}{3} R S^{e}$,
$G I_{\mathrm{Tc}}=\sum_{e_{\mathrm{c}}} G^{e} \cdot l^{e} \cdot t^{e} \cdot\left(\frac{\bar{u}_{j}-\bar{u}_{i}}{l^{e}}+d_{\mathrm{T}}^{e}\right)^{2} \cdot R S^{e}$ and

$G I_{\mathrm{T}}=G I_{\mathrm{To}}+G I_{\mathrm{Tc}}$.

Warping stiffness is calculated using the expression:

$E I_{\mathrm{W}}=\sum_{e} E^{e} \frac{l^{e} t^{e}}{3}\left(\bar{u}_{i}^{2}+\bar{u}_{i} \cdot \bar{u}_{j}+\bar{u}_{i}^{2}\right) \cdot R N^{e}$.

Using $G I_{\mathrm{T}}$ and $E I_{\mathrm{W}}$, the matrix $\mathbf{K}_{\mathbf{1 D}}$ for the $1 \mathrm{D}$ beam problem can be formed and relevant parameter distributions $\theta(x), \theta_{, x}(x), \theta_{, x x}(x), \theta_{, x x x}(x)$ can be determined for use in shear stress calculations. 


\section{Normal and secondary shear stresses due to restrained warping}

Restrained warping of a thin-walled beam will induce (a) normal stresses in a cross-section and (b) secondary shear stresses which will balance the longitudinally non-uniform distribution of normal stresses. This additional mechanism will influence the strain energy and the work of expression, so an iterative solution may be needed for greater accuracy.

Let $u(x, s)=\bar{u}(s) \cdot \theta_{x, x}(x)$ be the warping field in the cross-section calculated from the case of free torsion. Normal stresses are caused by restraining the warping, and vary along the $x$ axis. They are given by:

$$
\begin{aligned}
& \sigma_{x \mathrm{w}}=E \cdot \varepsilon_{x}=E \cdot \frac{\partial}{\partial x}\left(\bar{u}(s) \cdot \theta_{x, x}(x)\right) \text { or } \\
& \sigma_{x \mathrm{w}}^{i}=E^{e} \cdot \theta_{x, x x} \cdot \bar{u}_{i} \cdot R N^{e} .
\end{aligned}
$$

Let $u_{2}(x, s)$ be the secondary displacement field containing a displacement correction due to restrained warping. The total potential energy of a transverse strip consists of the internal energy generated from the fields $\epsilon_{2}$ and $\sigma_{2}$ (based on $u_{2}$ ) and the additional work done by the strip axial load $p_{x}$ on the secondary displacements $u_{2}$. If the change of $u_{2}$ along strip length $\Delta x$ is neglected, the total potential energy reads:

$\Pi=\sum_{e} \Pi^{e}=\sum_{e}\left[\frac{1}{2} \int_{V^{e}} G^{e}\left(\frac{\partial u_{2}}{\partial s}\right)^{2} \mathrm{~d} V-\Delta x \int_{S_{2}^{e}} \frac{\partial p_{x}}{\partial x} u_{2}(x, s) \mathrm{d} S\right]$.

The net external load $\Delta p_{x}$ due to restrained warping reads:

$\Delta p_{x}(x, s)=-\Delta \sigma_{x \mathrm{~W}}(x, s)=-E^{e} \cdot \bar{u}(s) \cdot \theta_{x, x x x}(x) \cdot R N^{e} \cdot \Delta x$,

and the total potential energy of the element, using the same shape functions as before, reads:

$$
\begin{aligned}
\Pi^{e} & =\frac{1}{2} \Delta x \cdot \mathbf{u}_{2}^{e \mathrm{~T}}\left(t^{e} \cdot G^{e} \cdot R S^{e} \int_{0}^{l^{e}} \mathbf{B} \mathbf{B}^{\mathrm{T}} \mathrm{d} s\right) \cdot \mathbf{u}_{2}^{e} \\
& +\Delta x \cdot \mathbf{u}_{2}^{e \mathrm{~T}}\left(\theta_{x, x x x} \cdot t^{e} \cdot E^{e} \cdot R N^{e} \int_{0}^{l^{e}} \mathbf{N} \mathbf{N}^{\mathrm{T}} \mathrm{d} s\right) \cdot \overline{\mathbf{u}}^{e}
\end{aligned}
$$

Minimization of the total potential energy with respect to the unknown displacement field $u_{2}$ leads to:

$\delta \Pi_{u_{2}}=0 \rightarrow \Delta x\left(\mathbf{K} \mathbf{u}_{2}+\mathbf{F}\right)=0 \rightarrow \mathbf{K} \overline{\mathbf{u}}_{2}=-\mathbf{F}$,

where: $\mathbf{K}$ is the global stiffness matrix as before, $\mathbf{u}_{2}$ is the global vector of unknown displacements $\mathbf{u}_{2}=\theta_{x, x x x} \cdot \overline{\mathbf{u}}_{2}, \mathbf{F}$ is the global load vector $\mathbf{F}=\theta_{x, x x x} \cdot \overline{\mathbf{F}}$. The element load and the secondary shear stresses (constant on element) read:

$\mathbf{F}^{e}=\theta_{x, x x x} \cdot R N^{e} \cdot E^{e} \cdot t^{e} \cdot l^{e}\left[\begin{array}{cc}1 / 3 & 1 / 6 \\ 1 / 6 & 1 / 3\end{array}\right]\left\{\begin{array}{l}\bar{u}_{i} \\ \bar{u}_{j}\end{array}\right\}=\theta_{x, x x x} \overline{\mathbf{F}}^{e} ;$

$\tau_{x s}^{k e^{(2)}}=G^{e} \frac{\partial u_{2}}{\partial s}=G^{e} \frac{u_{2 j}-u_{2 i}}{l^{e}}=G^{e} \frac{\bar{u}_{2 j}-\bar{u}_{2 i}}{l^{e}} \cdot \theta_{x, x x x}$.

The shear stress distribution can be calculated more accurately along the element (similar to the bending case) from the known element average stress $\tau_{x s}^{k e^{(2)}}$, the direction of shear stress flow, local element contribution $\tau_{2}(s)$ and its average $\tau_{2}^{k e}$ using expression $\tau_{x s}^{e^{(2)}}(s)=\tau_{x s}^{k e^{(2)}}-\tau_{2}^{k e}+\tau_{2}(s)$. After rearranging, it reads:

$\tau_{x s}^{e(2)}(s)=\left[G^{e} \frac{\bar{u}_{2 j}-\bar{u}_{2 i}}{l^{e}}\right.$

$\left.+\frac{E^{e} \cdot R N^{e}}{R S^{e}}\left(\left(\bar{u}_{i}+\frac{1}{2} \bar{u}_{j}\right) \frac{l^{e}}{3}-\bar{u}_{i} \cdot s-\frac{\bar{u}_{j}-\bar{u}_{i}}{2 l^{e}} s^{2}\right)\right] \cdot \theta_{x, x x x}$.

\section{Examples}

The first example is based on reports from the Advanced Subsonic Technology (AST) program. In the course of this program an experimental model of a composite wing box was made and tested (Fig. 3). [11] gives the loads carried by the hydraulic actuators which simulate the in-flight loading conditions.
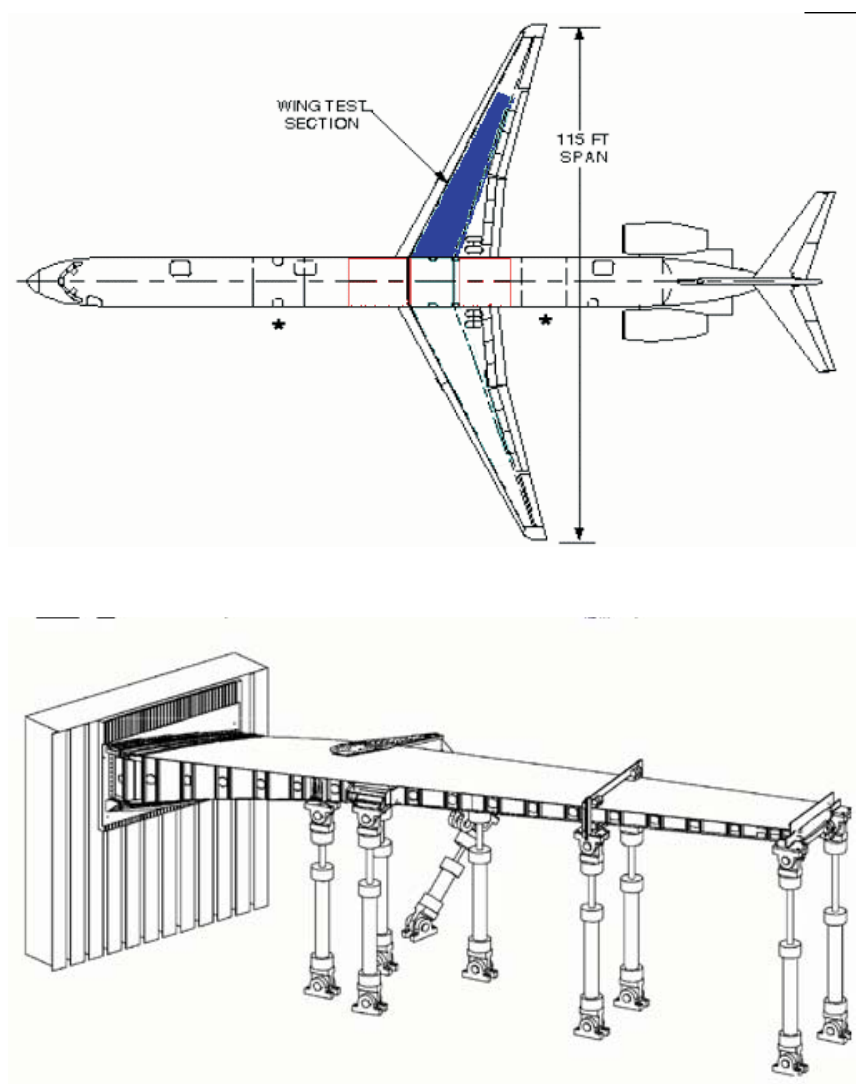

Fig. 3: MD 90 airplane and the test model of the wing box

The example shows a way of rapidly modeling and calculating the overall response of a similar metal wing box with linear behavior during the early stages of wing structural design. The analyzed wing box with reference to the AST box was shortened to 9.8 meters and modeled with high strength aluminum alloy 7075. The loads are decreased for the shortened wing box. The wing box is modeled by OCTOPUS 1D/2D combination.

Fig. 4 presents a model of the aluminum wing-box under modified $+2.5 \mathrm{G}$ loading conditions, and the unit response which needs to be multiplied by the values in parentheses to get the actual values for the considered load. The response components are decoupled to show the influence of each type 


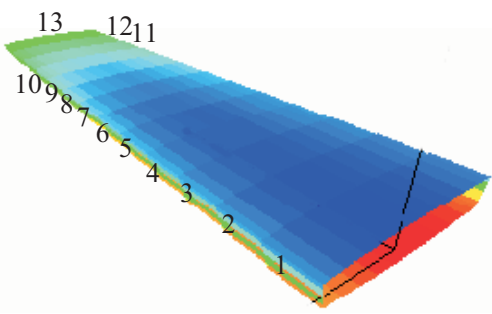

a) Metal wing box with 13 elements

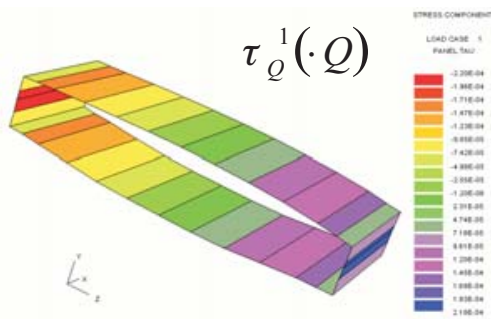

d) Shear stress (Bending)

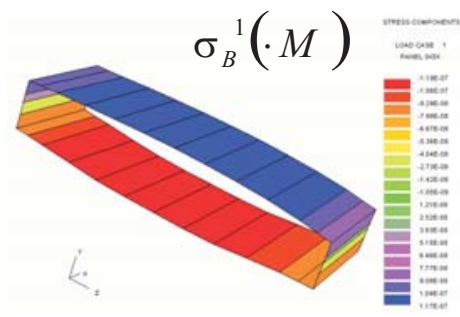

b) Normal stress (Bending)

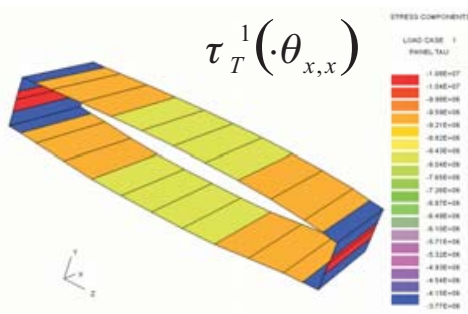

e) Shear stress (Torsion)

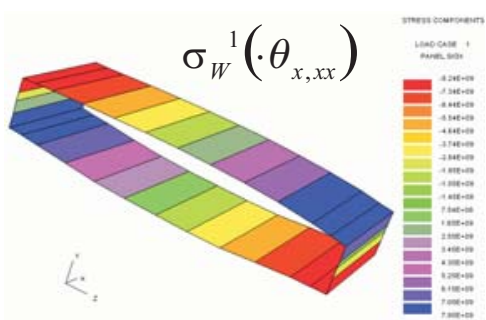

c) Normal stress (Restr. Warp.)

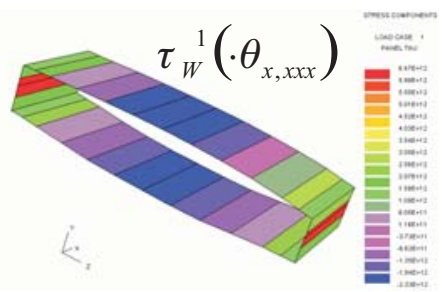

f) Shear stress (Restr. Warp.)

Fig. 4: Wing box model and unit responses of 1D FEM element 10 (between ribs 10 and 11)

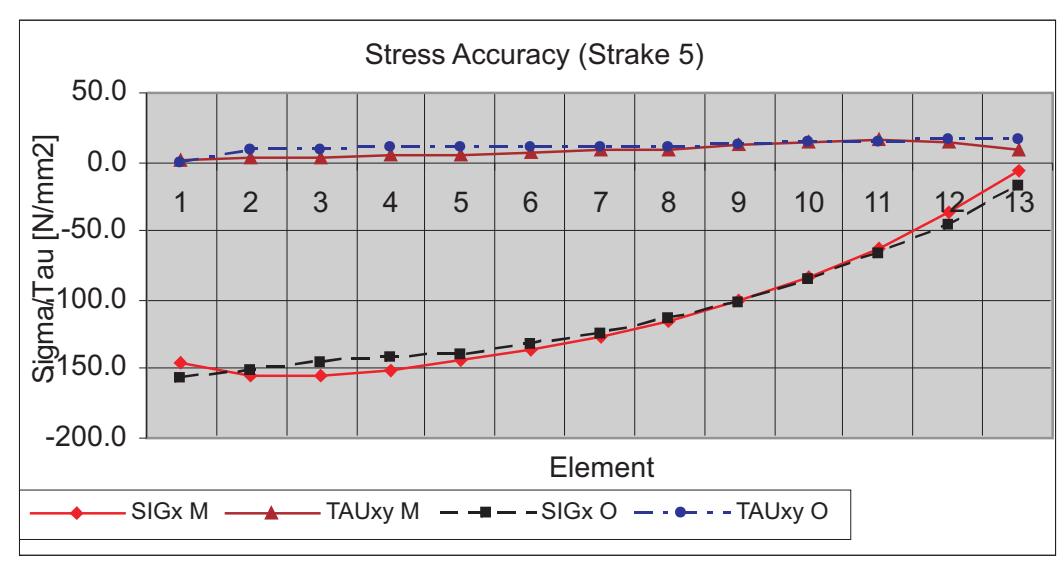

(a)

(b)

\begin{tabular}{|l|c|}
\hline \multicolumn{2}{|c|}{ 1D-Element 10} \\
\hline$y_{\mathrm{CG}}[\mathrm{mm}]$ & -14.5818 \\
\hline$z_{\mathrm{CG}}[\mathrm{mm}]$ & -337.763 \\
\hline$\varphi\left[{ }^{\circ}\right]$ & $7.14 \mathrm{E}-02$ \\
\hline$y_{\mathrm{SC}}[\mathrm{mm}]$ & -16.4946 \\
\hline$z_{\mathrm{SC}}[\mathrm{mm}]$ & -354.305 \\
\hline $\mathrm{A}\left[\mathrm{mm}^{2}\right]$ & 66270 \\
\hline $\mathrm{I}_{y}\left[\mathrm{~mm}^{4}\right]$ & $2.78 \mathrm{E}+10$ \\
\hline $\mathrm{I}_{z}\left[\mathrm{~mm}^{4}\right]$ & $1.66 \mathrm{E}+09$ \\
\hline $\mathrm{I}_{y z}\left[\mathrm{~mm}^{4}\right]$ & $3.26 \mathrm{E}+07$ \\
\hline $\mathrm{I}_{1}\left[\mathrm{~mm}^{4}\right]$ & $2.78 \mathrm{E}+10$ \\
\hline $\mathrm{I}_{2}\left[\mathrm{~mm}^{4}\right]$ & $1.66 \mathrm{E}+09$ \\
\hline $\mathrm{I}_{\mathrm{t}}\left[\mathrm{mm}^{4}\right]$ & $3.96 \mathrm{E}+09$ \\
\hline $\mathrm{Iw}_{\mathrm{w}}\left[\mathrm{mm}^{6}\right]$ & $2.85 \mathrm{E}+14$ \\
\hline $\mathrm{A}_{\mathrm{v}}\left[\mathrm{mm}^{2}\right]$ & 2412.777 \\
\hline $\mathrm{A}_{\mathrm{h}}\left[\mathrm{mm}^{2}\right]$ & 31405.07 \\
\hline
\end{tabular}

Fig. 5: (a) Stress accuracy across a wing span between Octopus (O) and Maestro (M), (b) cross-sectional properties of 1D-FEM element 10
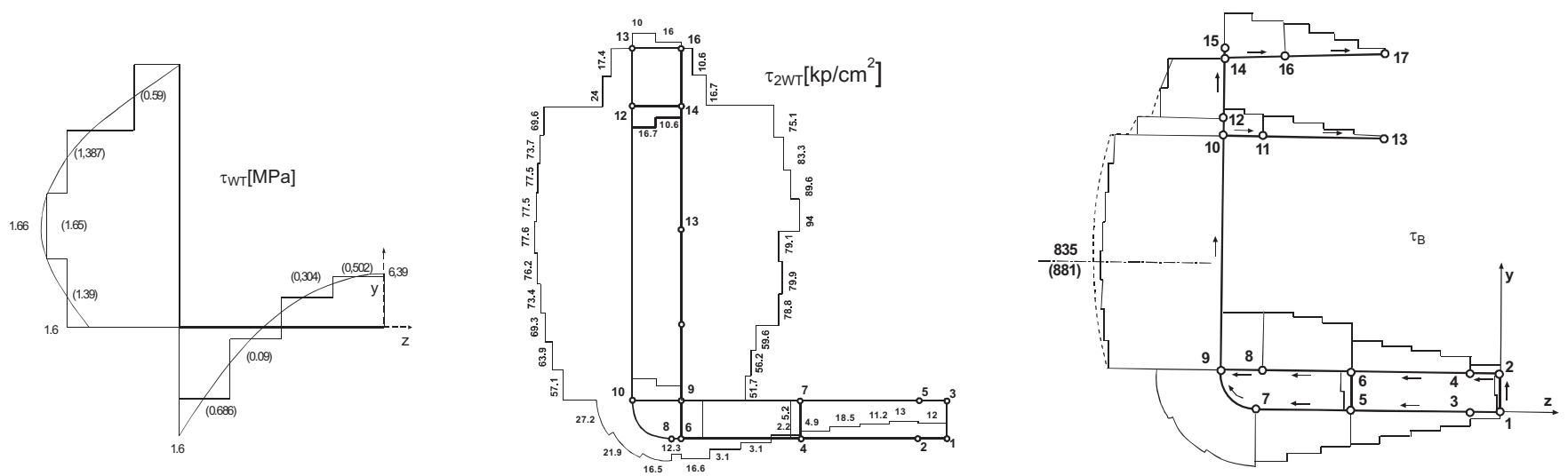

Fig. 6: (a) U-beam shear stress $\tau_{\mathrm{W}}$, (b) container ship shear stress $\tau_{\mathrm{W}}$, (c) general cargo ship shear stress $\tau_{\text {Bvert }}$ 
of load on the response. The cross section geometric characteristics obtained from 2D FEM and used in 1D FEM analysis are given in Fig. 5b.

The accuracy of the method is demonstrated in Figures $5 \mathrm{a}$ and 6 . Strake 5 of Fig. 5 a is located in the middle of the upper skin. Fig. 6 presents the application to the U-channel and two standard ship structures. It can be seen that the accuracy of the shear stress distribution based on FEM (constant per element) and analytical formulae (continuous line) in examples $6 \mathrm{a}$ and $6 \mathrm{c}$ is very good, even without parabolic correction. The verification examples are taken from [5].

\section{Conclusions}

A simple and practical method for calculating the primary response of monotonous structures (wings, ships, bridges) has been presented. All cross-section parameters are easily determined for complex stiffened thin-walled structures using a special FEM procedure. It could successfully replace classical, often cumbersome, analytical calculations. The method has been in constant use since 1980, applied to many real structures for concept design (in the OCTOPUS system) or as the generator of the force boundary conditions for partial 3D FEM models.

\section{References}

[1] Zanic, V., Das, P. K., Pu, Y., Faulkner, D.: "Multiple Criteria Synthesis Techniques Applied To Reliability Based design of SWATH Ship Structure." (Chapter 18). In: Integrity of Offshore Structures 5, (Faulkner, Das, Incecik, Cowling, editors), EMAS Scientific Publications, Glasgow, 1993, p. 387-415.

[2] Zanic, V., Rogulj, A., Jancijev, T., Bralic, S., Hozmec, J.: "Methodology for Evaluation of Ship Structural Safety." Proc. of $10^{\text {th }}$ Intl. Congress IMAM 2002, Crete, Greece, 2002, p. $54+$ CD.

[3] Zanic, V., Andric, J., Frank, D.: "Structural Optimization Method for the Concept Design of Ship Structures."
Proceedings of the $8^{\text {th }}$ International Marine Design Conference, Vol. 1; Papanikolau, A. D. (ed.), Athens, Greece, 2003., p. 99-110.

[4] Hughes, O. F.: Ship Structural Design. Wiley, 1983, SNAME 1992.

[5] Zanic, V.: "Calculation of Shear Flow in Cross-Section of Ship in Bending." (in Croatian). Proc. of Sorta Conference: Theory and practice in Shipbuilding, Split, Croatia, 1982.

[6] Zanic, V.: "Determinazione Degli Sforzi Principali -Flessione e Torsione-Sollo Scafo di una nave Applicando Particolari Elementi Finiti.” Technica Italiana (Rivista D'Ingegneria), No.3, 1985, p. 105-113.

[7] Prebeg, P.: Diploma thesis, University of Zagreb, 2003.

[8] MAESTRO Documentation, Proteus Eng. Stevensville, MD, USA, 2003.

[9] CREST Documentation, Croatian Register of Shipping, Split, Croatia, 2004.

[10] Hughes, O. F., Mistree, F., Zanic, V.: "A Practical Method for the Rational Design of Ship Structures." Journal of Ship Research, Vol. 24 (1980), No. 2, June 1980, p. 101-113.

[11] Karal, M.: AST Composite Wing Program - Executive Summary, NASA CR 2001-210650, 2001.

\footnotetext{
Vedran Zanic

phone: +385-1-6168122

fax: +385-1-6168399

e-mail: vedran.zanic@fsb.hr

Pero Prebeg

e-mail: pero.prebeg@fsb.hr

University of Zagreb

Faculty of Mechanical Engineering

and Naval Architecture

I. Lucica 5

10000 Zagreb, Croatia
} 